\title{
Gangliosides GM1 and GM3 in the Living Cell Membrane Form Clusters Susceptible to Cholesterol Depletion and Chilling ${ }^{\mathrm{D}}$
}

\author{
Akikazu Fujita, ${ }^{*}$ Jinglei Cheng, ${ }^{*}$ Minako Hirakawa, ${ }^{+}$Koichi Furukawa, ${ }^{\ddagger}$ \\ Susumu Kusunoki, ${ }^{+}$and Toyoshi Fujimoto*
}

\begin{abstract}
Departments of *Anatomy and Molecular Cell Biology and ‡Biochemistry II, Nagoya University Graduate School of Medicine, Nagoya 466-8550, Japan; and 'Department of Neurology, Kinki University School of Medicine, Osaka 589-8511, Japan
\end{abstract}

Submitted January 26, 2007; Revised March 6, 2007; Accepted March 20, 2007

Monitoring Editor: Jean Gruenberg

\begin{abstract}
Presence of microdomains has been postulated in the cell membrane, but two-dimensional distribution of lipid molecules has been difficult to determine in the submicrometer scale. In the present paper, we examined the distribution of gangliosides GM1 and GM3, putative raft molecules in the cell membrane, by immunoelectron microscopy using quick-frozen and freeze-fractured specimens. This method physically immobilized molecules in situ and thus minimized the possibility of artifactual perturbation. By point pattern analysis of immunogold labeling, GM1 was shown to make clusters of $<100 \mathrm{~nm}$ in diameter in normal mouse fibroblasts. GM1-null fibroblasts were not labeled, but developed a similar clustered pattern when GM1 was administered. On cholesterol depletion or chilling, the clustering of both endogenous and exogenously-loaded GM1 decreased significantly, but the distribution showed marked regional heterogeneity in the cells. GM3 also showed cholesterol-dependent clustering, and although clusters of GM1 and GM3 were found to occasionally coincide, these aggregates were separated in most cases, suggesting the presence of heterogeneous microdomains. The present method enabled to capture the molecular distribution of lipids in the cell membrane, and demonstrated that GM1 and GM3 form clusters that are susceptible to cholesterol depletion and chilling.
\end{abstract}

\section{INTRODUCTION}

Microdomains enriched with cholesterol and sphingolipids, or rafts, have been postulated to exist in the cell membrane (Simons and Ikonen, 1997). Domains showing a liquid-ordered state have been visualized in model membranes (Korlach et al., 1999; Dietrich et al., 2001a), but whether similar domains exist in the biological membranes of living cells, and what their basic properties would be, including size, life span and dynamics, are still under debate (Simons and Ikonen, 1997; Edidin, 2003; Munro, 2003; Kusumi et al., 2004; Mayor and Rao, 2004; Mukherjee and Maxfield, 2004). Recent results, obtained by single-particle tracking and fluorescent resonance energy transfer experiments, have suggested that rafts in normal unstimulated cells are extremely

This article was published online ahead of print in $M B C$ in Press (http:/ / www.molbiolcell.org/cgi/doi/10.1091/mbc.E07-01-0071) on March 28, 2007.

$\square$ The online version of this article contains supplemental material at MBC Online (http://www.molbiolcell.org).

Address correspondence to: Toyoshi Fujimoto (tfujimot@med.nagoya-u. ac.jp).

Abbreviations used: b-CtxB, biotinylated cholera toxin B; CI, confidence interval; CSR, complete spatial randomness; EM, electron microscopy; GAR-Fab5, colloidal gold (5-nm)-conjugated anti-rabbit IgG F(ab')2 fragment; PAG5, colloidal gold (5-nm)-conjugated protein $\mathrm{A} ; \mathrm{M} \beta \mathrm{CD}$, methyl- $\beta$-cyclodextrin; NND, nearest neighbor distance; PC, phosphatidylcholine; SDS-FRL, SDS-treated freezefracture replicas. small and may last for $<1 \mathrm{~ms}$ (Kenworthy et al., 2004; Kusumi et al., 2004; Sharma et al., 2004). Detergent-resistant membranes have often been regarded as an in vitro correlate of rafts, but detergents themselves have been found to cause domain formation artificially (Heerklotz, 2002). These results have thus posed questions regarding the true existence of rafts in living, nonstimulated cells. In addition, although microscopic identification of rafts has been attempted in a number of studies, putative raft molecules generally show diffuse distribution in the cell membrane without any concentration at the resolution of light microscopy. This result has been interpreted in several different ways, i.e., that rafts do not in fact exist, rafts are too small to be resolved by light microscopy, rafts occupy the majority of the membrane, or rafts do exist but may be disrupted by experimental procedures.

GM1 has been generally regarded as an authentic raft molecule, and cholera toxin B-subunit ( $\mathrm{CtxB})$ has been used to probe its distribution in many microscopic studies including FRET (Kenworthy et al., 2000; Nichols, 2003). However, because $\mathrm{CtxB}$ is a pentameric molecule that can bind to five GM1 molecules, binding of CtxB itself is likely to change the distribution of GM1 in the membrane as shown for membrane proteins that were cross-linked with antibodies (Mayor et al., 1994; Fujimoto, 1996). Some studies used specimens that were fixed before the CtxB labeling (Parton, 1994), but chemical fixatives are unlikely to preserve the in situ localization of membrane molecules, particularly lipids, and may even cause artifactual results (Jost et al., 1973; Chandler, 1984). 


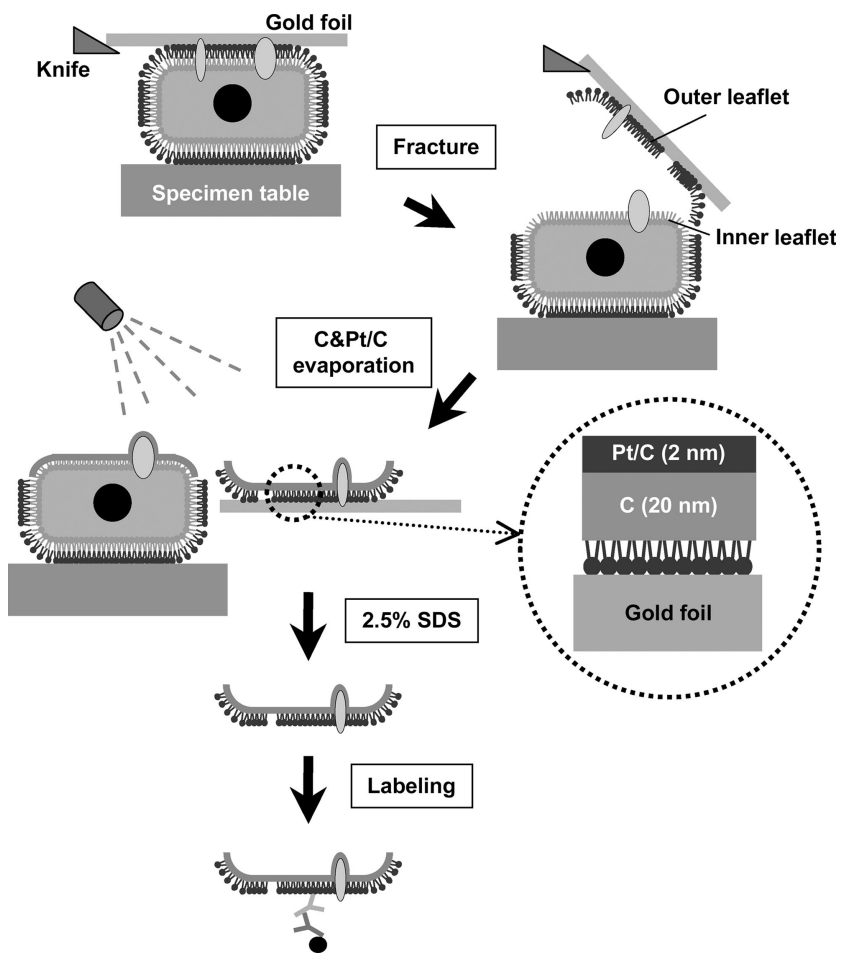

Figure 1. Outline of the SDS-FRL technique used in the present study. Cells cultured on thin gold foils were quick-frozen and freeze-fractured. Replicas were made by evaporation of carbon followed by platinum/carbon in most experiments. The replicas were treated with SDS and labeled with antibodies.

To avoid the possible artifacts caused by probe binding and chemical fixation, and also to take advantage of the high resolving power of electron microscopy (EM), we turned to immuno-EM using SDS-treated freeze-fracture replicas (SDSFRL) (Figure 1). In combination with rapid freezing, SDSFRL can immobilize membrane molecules physically and determine their localization in the nanometer range (Fujimoto, 1995). By use of this method, we wanted to examine whether putative raft lipids are distributed in clusters in the living cell membrane as postulated in the raft hypothesis.

In the present study, we labeled gangliosides GM1 and GM3 in the mouse fibroblast membrane, and we analyzed the result by spatial statistical methods in wide membrane areas. As a result, both GM1 and GM3 were found to form clusters in normal mouse fibroblasts, and their clustering became less obvious after cholesterol depletion. Contrary to the simplest raft hypothesis, dissolution of the cluster upon cholesterol depletion was not complete, and it showed regional heterogeneity; and, unexpectedly, a similar result was observed when cells were cooled on ice before rapid freezing. Additionally, clusters of GM1 and GM3 overlapped only partially, suggesting the presence of heterogeneous microdomains. These observations were discussed with reference to the findings obtained using other methodologies.

\section{MATERIALS AND METHODS}

\section{Antibodies and Probes}

Rabbit anti-GM1 antibodies were raised and affinity-purified as described previously (Kusunoki et al., 1996). Two different batches of antibodies were used in the present study, and they gave equivalent results. Mouse anti-GM3 antibody (clone GMR6; Seikagaku-Kogyo, Tokyo, Japan) and biotin-conjugated cholera toxin B-subunit (b-CtxB; Invitrogen, Carlsbad, CA) were pur- chased. Mouse anti-phosphatidylcholine (PC) antibody (JE-1) (Nam et al. 1990) was kindly donated by Dr. Masato Umeda (Kyoto University). Secondary antibodies conjugated with fluorochromes (Invitrogen), horseradish peroxidase (Pierce Chemical, Rockford, IL), and colloidal gold (BioCell, Cardiff, United Kingdom), and protein A coupled to colloidal gold (University Medical Center Utrecht, Utrecht, The Netherlands) were obtained from respective suppliers.

\section{Cells}

Fibroblasts were explanted from the dermis of wild-type mice and mice lacking the $\beta 1,4-N$-acetylgalactosamine-transferase gene (Takamiya et al., 1996). They were maintained in DMEM supplemented with $10 \%$ fetal calf serum, $50 \mathrm{U} / \mathrm{ml}$ penicillin, and $0.05 \mathrm{mg} / \mathrm{ml}$ streptomycin at $37^{\circ} \mathrm{C}$ under $5 \%$ $\mathrm{CO}_{2}, 95 \%$ air. The latter cells were referred to as GM1-null fibroblasts. To load GM1-null cells with exogenous GM1 (Sigma-Aldrich, St. Louis, MO), the cells were incubated in serum-free DMEM containing GM1 $(0.3,3$, or $30 \mu \mathrm{M})$ for $1 \mathrm{~h}$ at $37^{\circ} \mathrm{C}$ in the $\mathrm{CO}_{2}$ incubator. After incubation, the cells were washed twice with phosphate-buffered saline (PBS) and incubated with PBS containing $0.2 \%$ fatty acid-free bovine serum albumin (BSA) (Wako Pure Chemicals, Osaka, Japan) to remove GM1 loosely bound to the cell surface (Schwarzmann et al., 1983). For cholesterol depletion, cells were treated with $5 \mathrm{mM}$ methyl- $\beta$-cyclodextrin $(\mathrm{M} \beta C \mathrm{CD})$ in DMEM for $60 \mathrm{~min}$.

\section{Thin-Layer Chromatography (TLC) Immunoblotting and Dot Blotting}

Total lipids were extracted from cells by using chloroform/methanol, and glycosphingolipids were obtained by reverse phase chromatography with Sep-Pak C18 cartridge (Waters, Milford, MA) (Williams and McCluer, 1980). TLC was performed with high-performance TLC plates (Merck, Darmstadt, Germany) with a solvent system of chloroform $/$ methanol $/ 0.25 \% \mathrm{CaCl}_{2}(60$ : $35: 8)$. For staining by resorcinol, $0.5 \mu \mathrm{g}$ of gangliosides was loaded. For TLC immunoblotting, $0.25 \mu \mathrm{g}$ of gangliosides was developed on TLC plates, transferred to polyvinylidene difluoride membranes, and immunolabeled as described previously (Taki and Ishikawa, 1997). For dot blotting, glycosphingolipids were blotted on the nitrocellulose membrane. The signals of blotting were detected using the SuperSignal West Dura Extended Substrate (Pierce Chemical) according to the manufacturer's instruction.

\section{Immunofluorescence Microscopy}

Cells cultured on glass coverslips were fixed with buffered $4 \%$ formaldehyde, pretreated with $3 \%$ BSA, and incubated with antibodies or b-CtxB, followed by the fluorochrome-conjugated secondary reagents. In some experiments, unfixed cells were incubated with the primary probes on ice, fixed, and then labeled by the secondary reagents. The labeled samples were observed by an Axiophot2 microscope (Carl Zeiss, Jena, Germany) equipped with an AxioCam charge-coupled device camera using an Apochromat oil immersion $63 \times$ objective lens with a numerical aperture of 1.4. For quantification of filipin labeling intensity, focus was adjusted by rhodamine-phalloidin in the same specimen, and filipin fluorescence was pictured without observation by an identical microscope setting, and analyzed by ImageJ (National Institute of Health, Bethesda, MD).

\section{Quick-Freezing and Freeze-Fracture}

Cells grown on a small gold foil ( $\sim 4 \mathrm{~mm}^{2}$ in area; $20 \mu \mathrm{m}$ in thickness) were inverted upon prewarmed $10 \%$ gelatin on a gold-plated copper specimen table with the cell side down according to the metal sandwich method (Fujimoto and Fujimoto, 1997). The cell sandwich was slammed onto the copper block precooled to the liquid helium temperature $\left(-269^{\circ} \mathrm{C}\right)$ by using the rapid freezing apparatus HIF-4K (Hitachi High-Technologies, Tokyo, Japan).

For freeze-fracturing, the specimens were transferred to a cold stage of a Balzers BAF400 apparatus, and they were fractured at $-95^{\circ} \mathrm{C}$ and $\sim 2 \times 10^{-6}$ mbar. Replicas of the fractured membrane were made by electron-beam evaporation of platinum/carbon $(\mathrm{Pt} / \mathrm{C})$ and carbon $(\mathrm{C})$, and the replica thickness was controlled by a crystal thickness monitor. Three different evaporation protocols were tested: 1) Pt/C (2 nm) followed by C (20 nm), 2) C (20 $\mathrm{nm})$ followed by $\mathrm{Pt} / \mathrm{C}(2 \mathrm{~nm}), 3) \mathrm{C}(2 \mathrm{~nm})$ followed by Pt/C $(2 \mathrm{~nm})$, and then by $\mathrm{C}(20 \mathrm{~nm})$. After thawing, the replicas were immediately treated for $5 \mathrm{~min}$ in $2.5 \%$ SDS in PBS at $70^{\circ} \mathrm{C}$. They were adjusted to $50 \%$ glycerol and kept at $-20^{\circ} \mathrm{C}$ until labeling. Immunogold labeling was done as described previously (Fujimoto et al., 1996), and the specimens were observed with a JEOL 1200EX electron microscope operated at $100 \mathrm{kV}$.

\section{Statistical Analysis of Immunogold Labeling}

Electron micrographs were digitized with an image scanner. The $x-y$ coordinates of gold particles were obtained by Image Processing Tool Kit version 5 plug-in (Reindeer Graphics, Asheville, NC) for Adobe Photoshop version 6 (Adobe system, Mountain View, CA), and areas of $1 \mu \mathrm{m} \times 1 \mu \mathrm{m}$ chosen randomly were analyzed by Ripley's K-function (Ripley, 1979) by using a program provided by John Hancock (Prior et al., 2003). For significance tests, 
99\% confidence envelopes for complete spatial randomness (CSR) were generated from 100 Monte Carlo simulations. The frequency distribution of the nearest neighbor distance distribution was obtained by using the Image Processing Tool Kit.

\section{Measurement of Phospholipids Bound to Replicas}

The amount of phospholipids retained in SDS-treated C-Pt/C replicas was measured as described previously. Briefly, a suspension of small unilamellar PC liposomes was sandwiched between two thin copper foils, quickly frozen, freeze-fractured, and shadowed. One of the complementary replicas was washed in distilled water, and the other replica was treated with $2.5 \%$ SDS and washed with distilled water. The replicas were treated with $70 \%$ perchloric acid at $200^{\circ} \mathrm{C}$ for $2 \mathrm{~h}$, and the released phosphorus was measured (Zhou and Arthur, 1992).

\section{Biochemical Assay of Cholesterol}

The total lipids were extracted from cells by a mixture of hexane and isopropanol (3:2), and the amount of free cholesterol was measured by enzymatic fluorometric method as described previously (Heider and Boyett, 1978).

\section{On-Grid Model Experiment}

Colloidal gold ( $2 \mathrm{~nm}$; BioCell) conjugated with recombinant glutathione $S$ transferase (GST) was applied to Formvar-coated EM grids, blocked, and labeled with rabbit anti-GST followed by GAR-Fab5 or by PAG5. In other experiments, a dilute solution of recombinant GST was put on Formvarcoated grids and labeled by the same protocol.

\section{RESULTS}

\section{Antibody Specificity}

In enzyme-linked immunosorbent assay analysis with purified gangliosides, one of the two rabbit anti-GM1 antibodies used in our present study reacted with GM1 alone, whereas the other one also bound to GD1b and GA1. However, neither of these GM1 antibodies recognized GM2, GM3, GD1a, GD3, or GT1b (data not shown). In addition, by TLC blotting of gangliosides extracted from mouse fibroblasts, both of these antibodies showed positive reactivity only at the GM1 position and not GD1b or GA1 (Supplemental Figure 1). b-CtxB also reacted at the same position as the anti-GM1 antibodies. The monoclonal anti-GM3 antibody produced a band in cell extracts at the correct GM3 position and not GM1 (Supplemental Figure 1). The bands recognized by anti-GM1 and anti-GM3 antibodies were broader in the cell samples than that were for purified GM1 and GM3 molecules, but this is probably due to the presence of heterogeneous fatty acids, and/or the presence of glycolyl and acetylated forms of sialic acids in fibroblasts.

\section{Immunogold Labeling of Freeze-Fracture Replicas}

We previously showed that phospholipids are retained on SDS-treated freeze-fracture replicas and that they can be labeled by antibodies, but the labeling density was relatively low (Fujimoto et al., 1996). To explore different conditions that may facilitate the efficient labeling of GM1, we compared replicas prepared in three different ways. In replicas produced by the conventional evaporation method, e.g., $\mathrm{Pt} / \mathrm{C}(2 \mathrm{~nm})$ followed by C ( $20 \mathrm{~nm})$, labeling was found to be extremely low either by anti-GM1 antibody or by b-CtxB. The labeling intensity was improved, however, when the replicas were prepared by evaporating $\mathrm{C}$ before $\mathrm{Pt} / \mathrm{C}$. $\mathrm{C}(20$ $\mathrm{nm})-\mathrm{Pt} / \mathrm{C}(2 \mathrm{~nm})$ replicas and C (2 nm)-Pt/C (2 nm)-C (20 $\mathrm{nm}$ ) replicas gave equivalent results (Supplemental Figure 2 ). The retention of phospholipids in the SDS-treated replicas was not compromised by the change of the evaporation method: $82.6 \pm 13.0 \%(\mathrm{n}=3)$ phosphatidylcholine was retained in the C (20 nm)-Pt/C (2 nm) replicas, which was even better than the retention ratio obtained for the conventional $\mathrm{Pt} / \mathrm{C}(2 \mathrm{~nm})-\mathrm{C}(20 \mathrm{~nm})$ replicas (Fujimoto et al., 1996). Although the structural details became somewhat less de- fined in the C (20 nm)-Pt/C (2 nm) replica, we adopted this method in the present study due to its simplicity. Regardless of the procedure that was used for replica preparation, the labeling of GM1 was observed only in the E face, which represents the outer leaflet, and not in the $\mathrm{P}$ face, which represents the inner leaflet, or in the cytoplasm (Figure 3A, inset). Because the rabbit anti-GM1 antibodies gave significantly better labeling than $b-C t x B$, we used these antibodies in all of the subsequent EM experiments. The labeling efficiency of GM1 by the current method was calculated as described in Supplemental Table 1, and it was estimated that no less than $19.4 \%$ of GM1 in the original membrane was captured by immunogold labeling on the replicas.

The specificity of labeling in the replicas was confirmed using GM1-null cells (Takamiya et al., 1996). Replicas of those cells were devoid of labeling either by the anti-GM1 antibodies or by b-CtxB (Figure 2C). Significantly, however, positive labeling by both probes was observed when exogenous GM1 was added to the culture medium before freezing. The quantitative loading of GM1 was confirmed by dot blotting and immunofluorescence microscopy (Figure 2, A and B), and the density of replica labeling increased as the GM1 concentration in the medium was raised from 0.3 to 30 $\mu \mathrm{M}$ (Figure 2C). The distribution of the labeling in the GM1-loaded cells was indistinguishable from that in normal mouse fibroblasts. Detailed analysis of the labeling is described below. Because the current methodology precludes the observation of molecules outside the lipid bilayer, the aforementioned results also demonstrate that exogenous GM1 molecules are incorporated into the cell membrane and that they adopt a similar disposition to endogenous GM1.

\section{Distribution of GM1 in the Cell Membrane}

By use of the aforementioned technique, GM1 distribution in normal mouse fibroblasts was examined. Immunogold labeling gave apparently clustered distribution (Figure 3A). To obtain objective data, 50 areas of $1 \times 1 \mu \mathrm{m}$ were randomly chosen from samples obtained in more than three independent experiments, and the distribution patterns were assessed by point pattern analysis using Ripley's Kfunction (Ripley, 1977, 1979; Prior et al., 2003). When all the samples were compiled, the $L(r)-r$ curve was found to deviate most from the $99 \%$ confidence interval (CI) at a radius of $47.0 \mathrm{~nm}$ (Figure 3B). When individual samples were analyzed, the $L(r)-r$ curve showed a prominent peak except in a few cases (Supplemental Figure 3), and the peak size ranged from 32 to $68 \mathrm{~nm}$ (Figure 3C). We assumed that the basic cluster is in this size range (the size includes the arm length of the antibodies, which will be discussed later), and in subsequent experiments we classified the GM1 distribution patterns as "clustered" when the K-function was above the $99 \% \mathrm{CI}$ at more than one point below a $100-\mathrm{nm}$ radius. By this criterion, the GM1 labeling was clustered in all of the randomly chosen areas (50/50). The density of immunogold particles per unit area was found to be quite variable (Figure 4F), but GM1 clustering was observed irrespective of the labeling density.

The aforementioned result was obtained using rabbit antiGM1 as the primary antibody, and colloidal gold (5 nm)conjugated anti-rabbit IgG F(ab')2 fragment (GAR-Fab5) as the secondary probe. Because of the small size of the GM1 head group and the highly selective binding characteristics of the anti-GM1 antibody, it is unlikely that more than two primary antibodies bound to a GM1 molecule. In contrast, more than two GAR-Fab5 particles could bind to a primary antibody. However, we concluded that the clustering of GM1 labeling was not due to multivalency based on the 
A

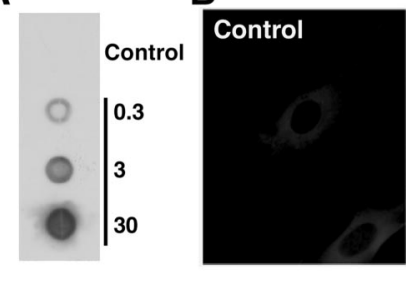

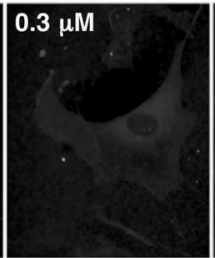
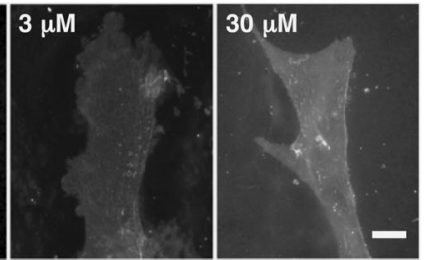

CAnti-GM1 Ab

Figure 2. Fibroblasts obtained from GM1-null mice were cultured with or without $0.3,3$, or $30 \mu \mathrm{M}$ GM1 for $60 \mathrm{~min}$. (A) Dot blot labeling by b-CtxB. (B) Fluorescence microscopy using b-CtxB, followed by fluorescein isothiocyanate-avidin. The addition of GM1 to the culture medium increases the GM1 content in GM1-null mouse fibroblasts. Scale bar, 10 $\mu \mathrm{m}$. (C) Freeze-fracture replicas were labeled by anti-GM1 antibody (top row), or by b-CtxB (bottom row). With either probe, labeling was not observed without GM1 loading, and labeling increased according to the amount of loading. Labeling of GM3 was observed without GM1 loading (inset). Tennanometer colloidal gold was used for labeling.
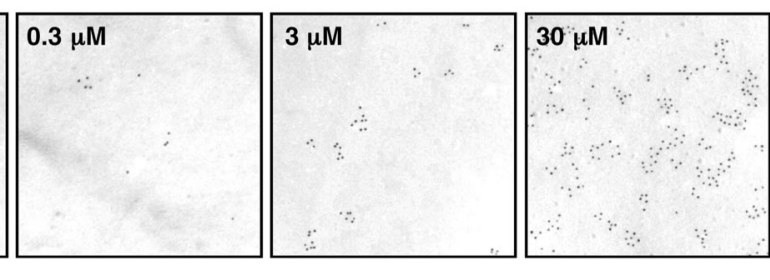

\section{b-CtxB}

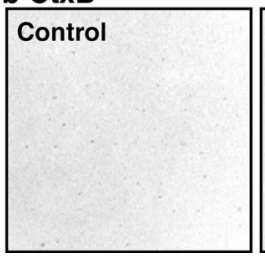

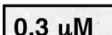

$0.3 \mu \mathrm{M}$
$3 \mu \mathrm{M}$

$30 \mu \mathrm{M}$ following results. First, a model experiment showed that two or three GAR-Fab5 particles could bind to an IgG molecule in 15.7 and $3.6 \%$ of the cases, respectively. When random point patterns were generated and the above-mentioned proportions of points were duplicated or triplicated, however, the resultant patterns did not show clustering as analyzed by Ripley's K-function (Supplemental Figure 4). Second, a very similar clustering was obtained when using colloidal gold (5 nm)-conjugated protein A (PAG5) as the secondary probe (Supplemental Figure 5, A and B). Only one PAG5 particle should bind to an IgG molecule, and the result of the model experiment was consistent with this principle (Supplemental Figure 4).

We next analyzed the entire area of randomly chosen cells to examine possible local heterogeneity within a single cell. Replicas were often disrupted within the cellular boundary, but areas of $145 \mu \mathrm{m}^{2}$ (ranging from 55 to $327 \mu \mathrm{m}^{2}$ ) could be observed on average for each cell. From this analysis, $70 \%$ of the cells showed only a clustered distribution throughout their surface, but the remainder showed small areas of random distribution (Figure 4D). An example of a whole cell profile and local $L(r)-r$ curves is shown in Supplemental Figure 6.

\section{Effects of Cholesterol Depletion and Low Temperatures on GM1 Clustering}

We next examined distribution of GM1 in mouse fibroblasts after depleting cholesterol to disrupt rafts. In cells treated with $5 \mathrm{mM} \mathrm{M} \beta C D$ for $60 \mathrm{~min}$, the free cholesterol content was reduced considerably (Supplemental Figure 7). In these cells, $28 \%(14 / 50)$ of the areas showed random GM1 distribution, but $72 \%(36 / 50)$ still showed clustering (Figure 4C). Essentially, the same result was obtained when PAG5 was used for labeling (Supplemental Figure 5, B and C). Analysis of the compiled data also showed clustering (Figure 4A), but when individual samples were assessed, the peak of the $L(r)-r$ curves was clearly lower, broader, and less distinct compared with the control sample (Supplemental Figure 3).
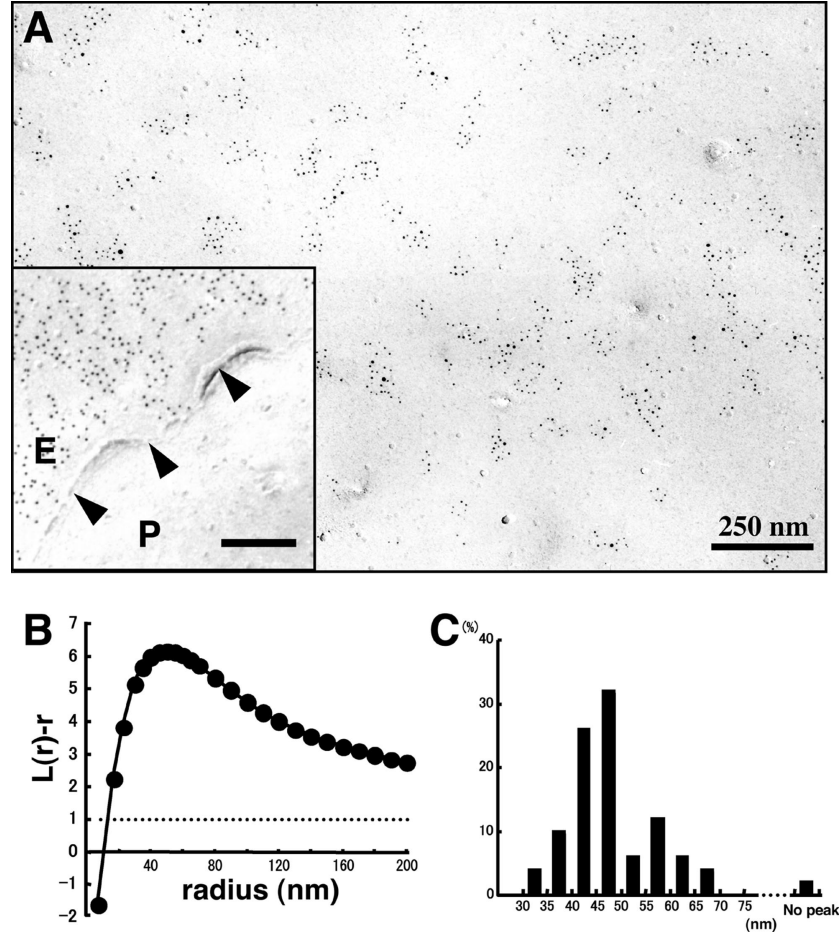

Figure 3. Labeling of freeze-fracture replicas of normal mouse fibroblasts by rabbit anti-GM1 antibody and colloidal gold (5-nm)conjugated anti-rabbit IgG F(ab')2 fragment (GAR-Fab5). (A) GM1 labeling by 5 -nm colloidal gold particles was detectable as clusters in the $\mathrm{E}$ face of the freeze-fractured plasma membrane. Inset, the $\mathrm{P}$ face was unlabeled. The cell boundary is marked by arrowheads. Ten-nanometer colloidal gold was used for labeling in this sample. (B) Fifty areas $(1 \times 1 \mu \mathrm{m})$ were randomly photographed, and the gold point patterns were analyzed by Ripley's K-function. The mean $L(r)-r$ curve showed maximal deflection from CSR $(99 \%$ CI is shown by a dotted line) at a 47.0-nm radius. (C) Radii of maximal deflection for 50 sample areas ranging from 32 to $68 \mathrm{~nm}$, with only one area showing no apparent peak. 
A

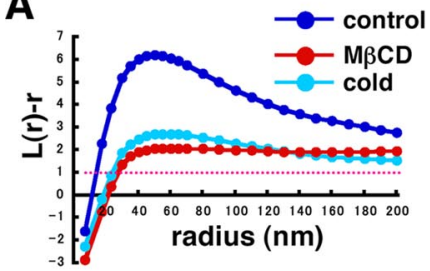

C $\square$ Clustered $\square$ Random

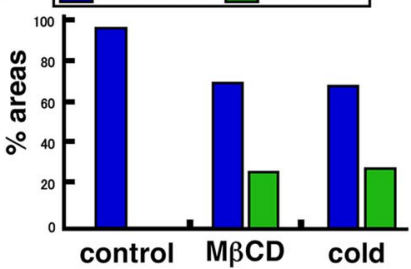

F

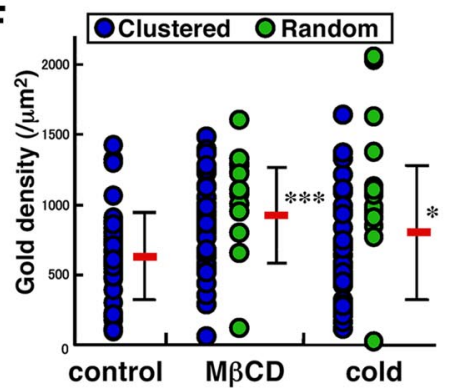

B
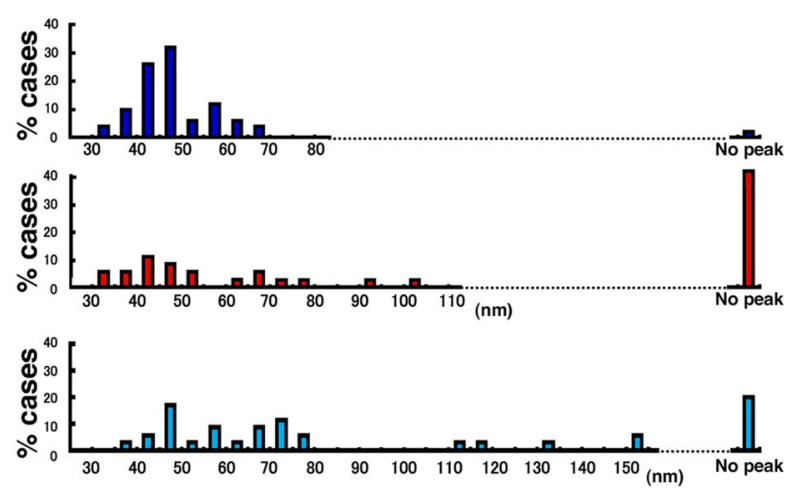

G

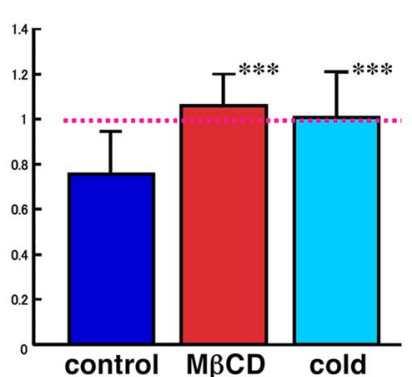

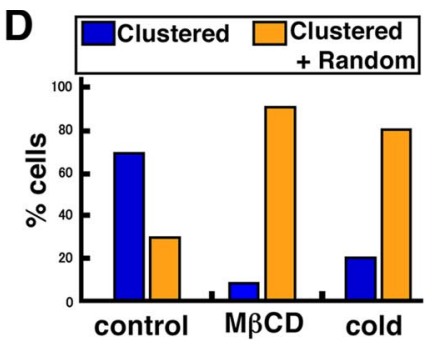

E

H

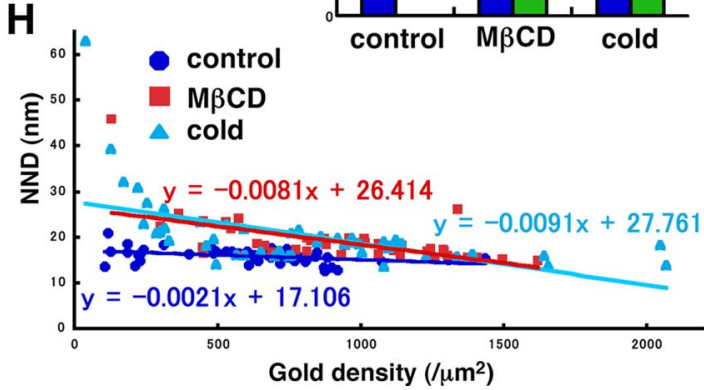

Figure 4. Analysis of GM1 distribution in normal mouse fibroblasts under three different conditions: control, cholesterol depletion, and incubation on ice for $30 \mathrm{~min}$. (A) Mean $L(r)-r$ curves. The pooled data show clustering even after cholesterol depletion or chilling, but deviation from CSR was considerably smaller than the control. (B) Radii of maximal deflection in 50 areas. $L(r)-r$ curves without any peak below $r=200 \mathrm{~nm}$ increased after either treatment. (C) Classification based on K-function analysis. Areas showing more than one point above the $99 \%$ CI below $r=100 \mathrm{~nm}$ were regarded as clustered. (D) Classification of 10 randomly chosen cells. The entire area in each cell was analyzed. Cells were classified by whether they showed clustered areas only or both clustered and random areas. (E) NND analysis. NND values increased significantly after cholesterol depletion or chilling, compared with control cells (Student's $t$ test; ${ }^{* *} \mathrm{p}<0.005,{ }^{* * *} \mathrm{p}<0.001$ ). (F) The average labeling density showed a wide range for each sample and increased significantly after cholesterol depletion or chilling (Student's $t$ test; ${ }^{*} \mathrm{p}<0.05,{ }^{* * *} \mathrm{p}<0.001$ ). (G) NND normalized to the value expected for random distribution. Only the control sample showed a value significantly $<1\left(^{* * *} \mathrm{p}<0.001\right)$. $(\mathrm{H})$ Correlation of NND and the average labeling density. The dependence of NND upon the labeling density was far less in the control than in the treated samples.

In cholesterol-depleted wild-type mouse fibroblasts, the clustered and random distribution patterns of GM1 coexisted in $>90 \%$ of the cells (Figure $4 \mathrm{D}$ ). As shown in representative cases (Figure 5 and Supplemental Figure 6), the two different patterns were often observed side by side in narrow areas of the membrane. The result confirmed that the clustering of GM1 was not caused artifactually by the experimental procedures after freezing.

With the assumption that a low temperature would increase the raft area, and thus induce a further GM1 clustering, we kept mouse fibroblasts on ice for $30 \mathrm{~min}$ before rapid freezing. In contrast to the assumption, however, the distribution of the GM1 labeling in those cells showed changes that were very similar to those observed in cholesteroldepleted cells. The areas showing both clustered and random GM1 patterns were $70 \%(35 / 50)$ and $30 \%(15 / 50)$, respectively (Figure $4 \mathrm{C}$ ). Individual samples also revealed a similar tendency, and the $L(r)-r$ curves generally became less distinct and broader than the control (Supplemental Figure 3 ). The coexistence of both clustered and random distribution patterns of GM1 in these cells was also observed in $80 \%$ of cases (Figure 4D). The changes caused by the cold were not likely to be caused by energy depletion because treatment with $10 \mathrm{mM}$ sodium azide and $10 \mathrm{mM}$ 2-deoxyglucose for $30 \mathrm{~min}$, which inhibited endocytosis of CtxB completely, did not affect the GM1 distribution (data not shown).

Cholesterol depletion and low temperatures caused similar changes in two other indices: both the nearest neighbor distance (NND) and the average labeling density values increased significantly (Figure 4, E and F). The average NND, normalized to the value expected for random distribution, was significantly $<1$ in the control cells, but it became close to 1 after either cholesterol depletion or chilling (Figure 4G). Most significantly, the NND was virtually independent of the labeling density in control cells, whereas the NND decreased as the labeling density increased in cells after cholesterol depletion or chilling (Figure $4 \mathrm{H}$ ). These data strongly indicate that GM1 in control mouse fibroblasts is clustered and that it becomes less so after cholesterol depletion or chilling.

The changes caused by cholesterol depletion and the cold were observed similarly in GM1-null cells preloaded with 10-15 $\mu \mathrm{M}$ GM1. The peak of the $L(r)-r$ curves became lower (Figure 6A), the frequency of random areas increased (Figure 6B), and the average labeling density increased (Figure 6C). Cholesterol depletion caused a more drastic change in the GM1-loaded cells than in the normal mouse fibroblasts. The reason is not clear, but some difference in the fatty acid composition between exogenous GM1 derived 


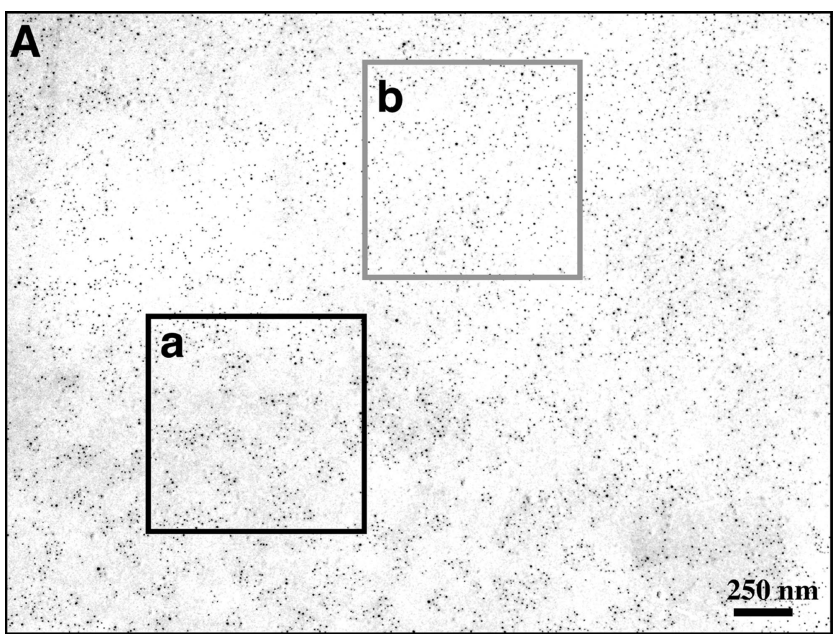

B

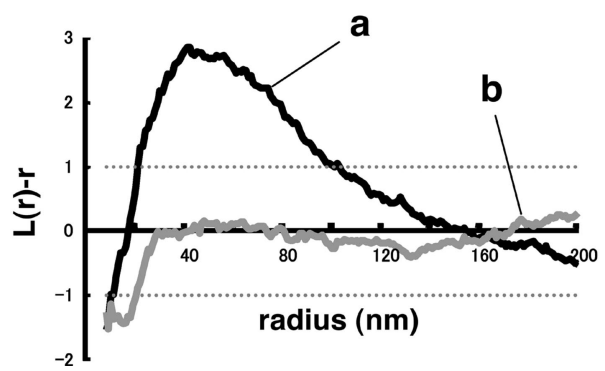

Figure 5. GM1 labeling in mouse fibroblasts treated with $5 \mathrm{mM}$ $\mathrm{M} \beta C D$ for $60 \mathrm{~min}$. (A) The labeling shows a marked regional heterogeneity in most cells. (B) The gold point patterns of adjacent areas in A show clustered (a) and random (b) distributions, respectively.

from bovine brain and GM1 expressed endogenously in mouse fibroblasts may be involved.

As a control, PC was labeled by SDS-FRL. Probably because the epitope of the anti-PC antibody includes a hydrophobic portion of PC that is located deeper in the lipid bilayer (Nam et al., 1990), the antibody can only bind to a certain population of PC molecules, and the labeling does not occur homogenously even on liposome replicas (see figure 2A of Fujimoto et al., 1996). A similar patchy labeling pattern was observed in the mouse fibroblast (Figure 7A). In contrast to GM1 (Figure 3) and GM3 (see below), the distribution of the PC labeling was not affected by cholesterol depletion, whereas chilling caused reduction of clustering (Figure 7B). Because of the unidentified binding characteristics of the anti-PC antibody, definite interpretation of the result is not possible, but the result showed that the distributional changes do not occur in the same manner in membrane lipids.

\section{Distribution of GM3 in Relation to GM1}

We next examined distribution of another ganglioside, GM3, by the same technique. GM3 also showed a clustered pattern in normal mouse fibroblasts (Figure 8). In comparison with GM1, the $L(r)-r$ curve of the GM3 labeling did not show an evident peak (Figure 8A), indicating that the size of the GM3 cluster was highly variable and generally larger than that of the GM1 cluster. We confirmed that the indirect immunolabeling protocol, i.e., mouse immunoglobulin M (IgM) followed by colloidal gold-conjugated anti-mouse Ig antibody, did not cause prominent clustering by a model experiment similar to Supplemental Figure 4 (data not shown). In cells depleted of cholesterol by $M \beta C D$, the clustering of GM3 decreased significantly (Figure $8 \mathrm{~B}$ ). According to the criterion used for GM1, 26.7\% (8/30) of the area showed random distribution, and in the rest of the cases, the $L(r)-r$ curve was out of the CI range (Figure $8 \mathrm{C}$ ). But as in the case of GM1, analysis of the individual areas showed that the $L(r)-r$ curves became generally lower in cholesterol-depleted cells even when they were classified as "clustered" by the criterion used for GM1 (Supplemental Figure 8). These results indicated that the clustering of GM3 is also related to the cholesterol content of the plasma membrane.

The relationship between the GM1 and GM3 clusters in normal mouse fibroblasts was examined by double labeling by using colloidal gold particles of two different diameters. By use of the bivariate K-function, two different patterns were observed. In a relatively limited number of areas (13.3\%), GM1 and GM3 were observed to make coclusters,
Figure 6. Changes observed after cholesterol depletion and chilling in normal mouse fibroblasts were also observed in GM1-null fibroblasts loaded with exogenous GM1. (A) The mean $L(r)-r$ curve shows a decrease of clustering after cholesterol depletion by $\mathrm{M} \beta \mathrm{CD}$ or after chilling. The change was more drastic by cholesterol depletion than by chilling. ( $\mathrm{B}$ and C) The proportion of areas showing random distribution as well as the average labeling density also increased significantly by cholesterol depletion or chilling.
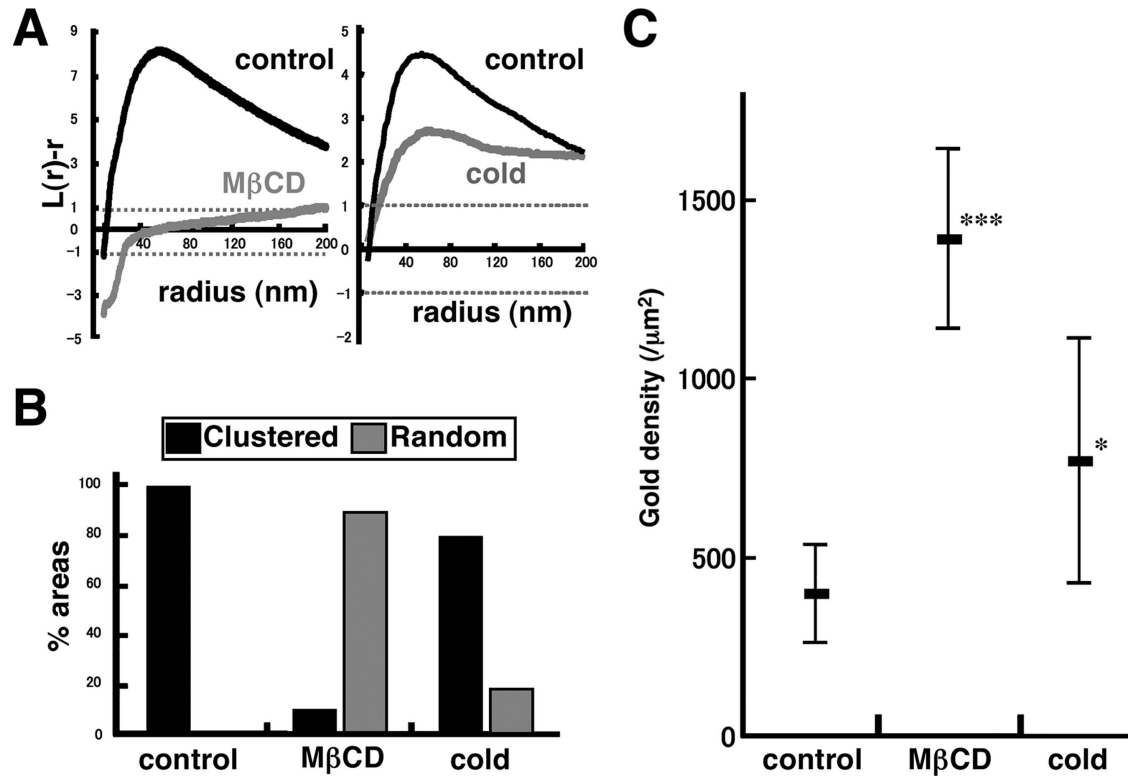
A

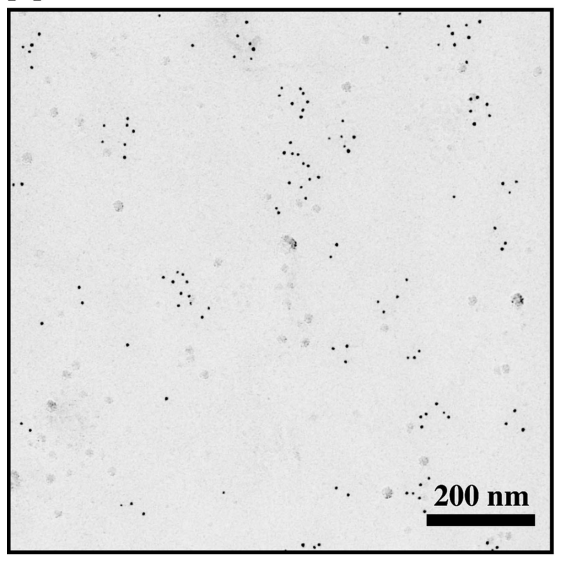

B

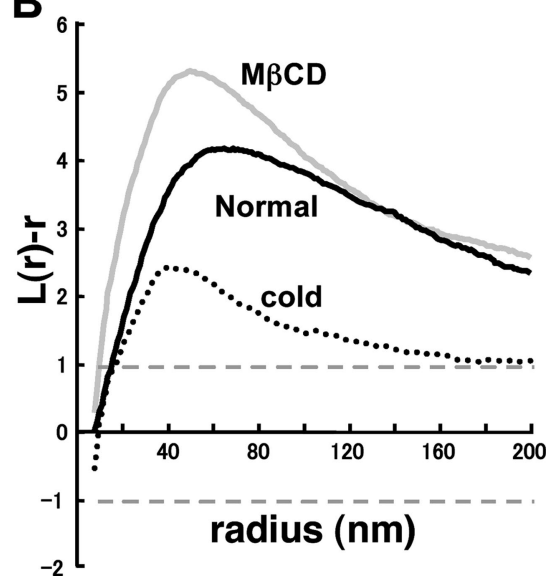

Figure 7. Distribution of PC labeled by mouse anti-PC antibody (IgM) and colloidal gold (5-nm)-conjugated goat anti-mouse Ig antibody. (A) The labeling of PC in the normal mouse fibroblast was observed as clusters in the E face, which was similar to that seen in liposomes and in other cell types (Fujimoto et al., 1996). (B) The K-function analysis of 10 areas $(1 \times 1 \mu \mathrm{m})$ showed that the labeling was clustered in the normal mouse fibroblast. The clustering was observed similarly in cells depleted of cholesterol, but it decreased in chilled cells. whereas in most areas (86.7\%), the GM1 and GM3 clusters were segregated and formed independently (Figure 9, A and B). When coclustering was observed, GM1 clustering was less discrete than that observed by single labeling, which was evident in the $L(r)-r$ curve of the univariate K-function analysis (data not shown). This difference was likely caused by steric hindrance between the anti-GM1 and anti-GM3 antibodies binding to overlapping areas.

\section{DISCUSSION}

\section{Methodological Considerations}

SDS-FRL has several advantages for analyzing the distribution of membrane molecules in the submicrometer range. By freezing live cells quickly to the liquid helium temperature, the molecular motion in the cell should stop immediately. Phase separation may occur in membranes that were cooled slowly, but it was prevented by this quick-freeze method (Thompson et al., 1985). In their pioneering article, Heuser and his colleagues showed that the surface of a muscle piece became frozen within $1 \mathrm{~ms}$ after the impact with the liquid helium-cooled copper block (Heuser et al., 1979). They later found that the freezing rate is at least 10 times faster than that (Heuser, personal communication). Although details in the present experiment were different from their experiment, the cell membrane of thin culture cells should be frozen at a better cooling rate than the muscle piece. Further sophistication in the measurement of the freezing rate is required to assess the time and spatial resolution of this method precisely.

During the freeze-fracture procedure, the specimens were kept frozen below $-100^{\circ} \mathrm{C}$, and the fractured membrane half was coated by evaporation of $\mathrm{C}$ and $\mathrm{Pt} / \mathrm{C}$ to immobilize membrane molecules in situ. The physical fixation provided by the combination of quick-freezing and freeze-fracturing contrasts with chemical fixation by aldehydes in many respects. Aldehydes have only limited reactivity with membrane lipids and could even cause redistribution of membrane proteins by a cross-linking effect (Kusumi and Suzuki, 2005). More than several seconds are required for the aldehyde fixation to complete, and even after fixation, membrane molecules retain their two-dimensional mobility (Jost et al., 1973; Chandler, 1984). Thus, in specimens fixed by formaldehydes, binding of probes, especially multivalent probes, could cause redistribution of membrane molecules (Mayor et al., 1994). Although glutaraldehyde was shown to prevent gross redistribution of membrane proteins (Mayor et al., 1994), it is unlikely to stabilize membrane lipids in the nanometer range. The membranes stabilized in replicas preclude the possibility of such artifacts caused by probes.

Compared with the conventional method that evaporates $\mathrm{Pt} / \mathrm{C}$ before $\mathrm{C}$, the labeling of gangliosides was improved drastically by evaporating $\mathrm{C}$ before $\mathrm{Pt} / \mathrm{C}$. A similar effect has been reported for some membrane proteins (Hagiwara et
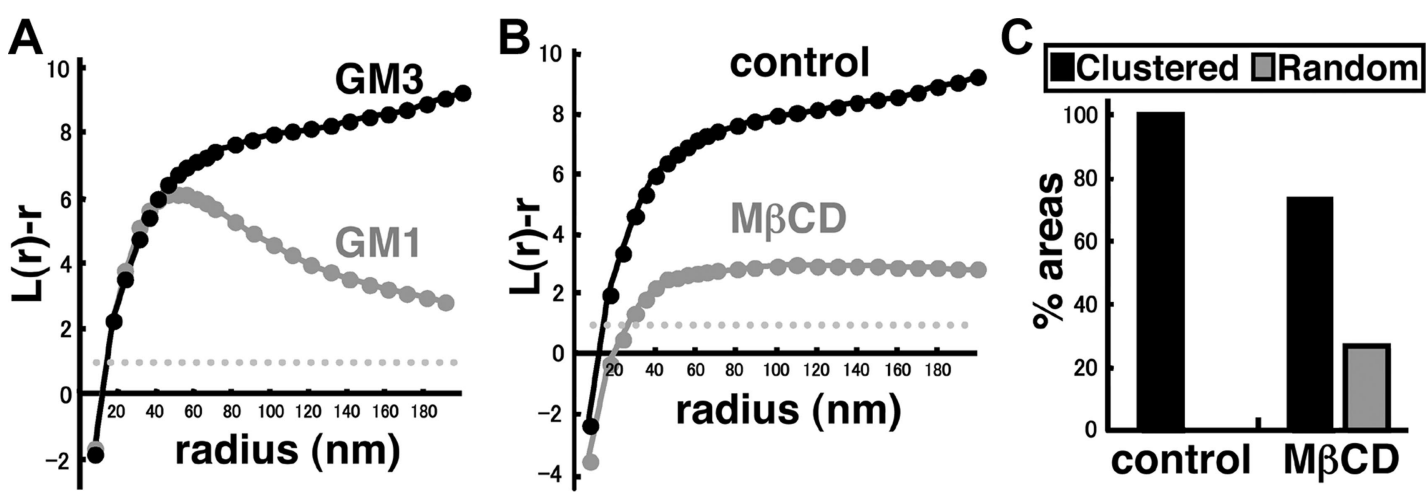

Figure 8. GM3 labeling in normal mouse fibroblasts. (A) Thirty randomly chosen areas $(1 \times 1 \mu \mathrm{m})$ were analyzed by Ripley's K-function. In contrast to GM1, the mean $L(r)-r$ curve of GM3 did not show any apparent deflection below $r=200 \mathrm{~nm}$. (B) The mean $L(r)-r$ curve became lower after cholesterol depletion. (C) Classification of the 30 areas according to K-function analysis. Cholesterol depletion caused an increase in the number of areas with random distribution. 

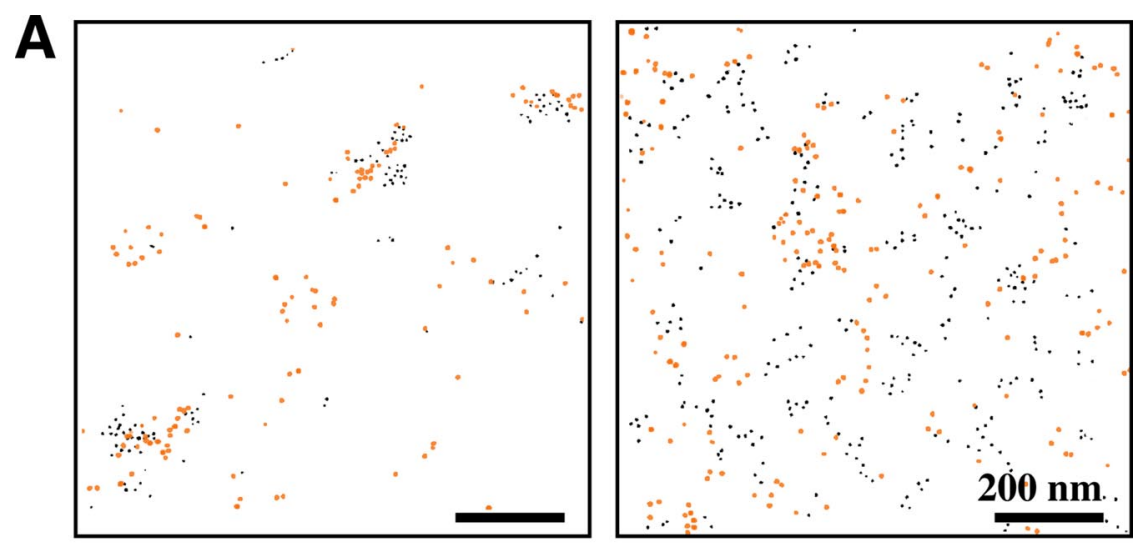

Figure 9. Double labeling of GM1 and GM3 in normal mouse fibroblasts. (A) GM1 and GM3 were marked with colloidal gold particles of different sizes, which were colored artificially: GM1, black; GM3, orange. (B) Analysis of the two areas shown in A by using a bivariate K-function. GM1 and GM3 were found to be coclustered in the left sample, but they segregated from each other in the right sample. Coclustering as shown in the left graph was seen only in $13.3 \%$ of the cases.

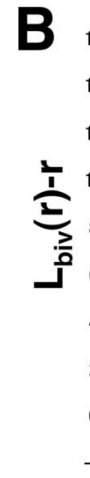

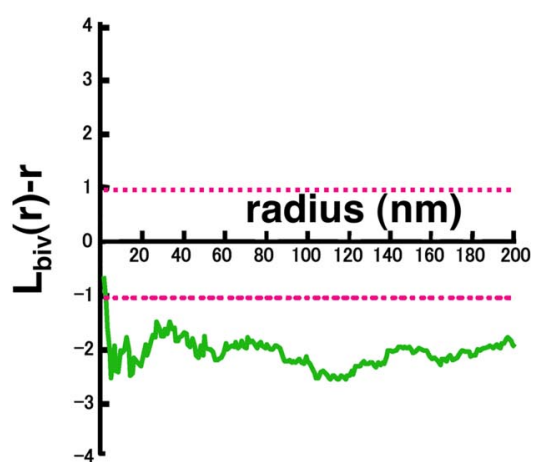

al., 2005). The reason for this improvement is not fully clear, but compared with $\mathrm{Pt} / \mathrm{C}$, pure $\mathrm{C}$ casting may allow the molecules to retain some flexibility, which may facilitate their interaction with probes. Additionally, the use of antibodies improved the GM1 labeling in SDS-FRL. CtxB worked well for immunofluorescence microscopy, but it labeled the replica to a much lesser extent than the anti-GM1 antibodies. For highaffinity binding of pentameric CtxB molecules, GM1 molecules and surrounding lipids may need to be rearranged properly, and this probably occurs frequently in the fluid membrane. The relatively low labeling by SDS-FRL may have resulted because molecules in the replica are immobilized, and they do not take an appropriate arrangement very often.

\section{GM1 Clustering in the Cell Membrane}

The GM1 labeling formed clusters with an average radius of $47.0 \mathrm{~nm}$ in control mouse fibroblasts. The average labeling density ranged from $<100$ to $>1400$ particles $/ \mu \mathrm{m}^{2}$, but clusters of similar sizes were observed irrespective of the GM1 density. In our present method, two layers of probes, either a whole IgG molecule and a $\mathrm{F}\left(\mathrm{ab}^{\prime}\right) 2$ portion (GAR-Fab5) or a whole IgG and protein A (PAG5), were intercalated between colloidal gold $(5 \mathrm{~nm})$ and the antigen. Using a model experiment, the spacer distances were estimated as $16.3 \pm$ $4.1 \mathrm{~nm}$ (GAR-Fab5) and $16.1 \pm 5.3 \mathrm{~nm}$ (PAG5) (Supplemental Figure 10). This lead to a calculated size of the GM1 cluster of $\sim 61 \mathrm{~nm}$ in diameter, which roughly corresponds to that which was estimated by several studies for glycosylphosphatidylinositol (GPI)-anchored protein clusters (Friedrichson and Kurzchalia, 1998; Varma and Mayor, 1998; Pralle et al., 2000), but it is larger than the estimate obtained in more recent studies by using sophisticated biophysical techniques (Kusumi et al., 2004; Sharma et al., 2004). Ras proteins were also shown to make smaller clusters by im-
muno-EM of mechanically detached membranes (Plowman et al., 2005). The size difference of the clusters is not surprising, however, because GPI-anchored proteins were shown to behave differently from GM1 in several instances (Schnitzer et al., 1995; Simons et al., 1999; Dietrich et al., 2001b). It is also likely that the Ras cluster in the inner leaflet does not coincide with that of GM1 in the outer leaflet. SDS-FRL should be able to study the distribution of those proteins and to compare the cluster sizes directly.

After cholesterol depletion or incubation of cells on ice, GM1 labeling became less clustered, and areas showing random distribution increased significantly. In addition, cholesterol depletion and chilling increased the average labeling density as well as the NND. The increase of the labeling density could reflect a real increase of GM1 molecules in the plasma membrane, but the change of the aforementioned three parameters together, and above all, the virtual independence of NND values from the labeling density in control cells (Figure $4 \mathrm{H}$ ), is more likely to suggest a dispersion of GM1 clusters by cholesterol depletion or by chilling. That is, GM1 molecules densely packed in control cells are probably not labeled efficiently due to steric hindrance, but after cholesterol depletion or chilling, they may be dispersed and more GM1 molecules may become accessible to antibodies. This possibility also suggests that the density of GM1 molecules in the cluster is underestimated in immunogold labeling.

An important question that arises from our current data is the very nature of these GM1 clusters. The clusters may be explained by the presence of rafts, or liquid-ordered (lo) domains, and by preferential partitioning of GM1 in the domains. The results of our chilling experiments would not seem consistent with this supposition, because low temperatures are expected to increase more ordered domains, 
whereas cholesterol depletion should disrupt lo domains (Simons and Ikonen, 1997; Gaus et al., 2003). However, as the temperature is decreased, the lo domain should become the dominant or percolating phase, and GM1 and other raftphilic molecules that are confined to small nonpercolating lo domains at the ambient temperature may adopt a dispersed distribution in the expanded lo domains (Meder et al., 2006). Hence, the dispersed GM1 distribution after chilling can be interpreted to reflect the larger lo domain, or an increase of raft areas. This contrasts with the similarly dispersed GM1 distribution after cholesterol depletion, which may be caused by an increase of the liquid-disordered domains, or disruption of rafts.

However, the incomplete dissolution of the GM1 cluster and the marked regional heterogeneity upon cholesterol depletion suggest that preferential partitioning in the ordered membrane may not explain the whole phenomenon. In this context, it is notable that several studies showed formation of GM1-rich microdomains within the ordered phase of model membranes (Vie et al., 1998; Yuan and Johnston, 2000; Yuan et al., 2002), suggesting that glycolipids are capable of self-organization (but please note that other studies failed to observe the clustering of charged gangliosides; Thompson et al., 1985; Wang and Silvius, 2003). Furthermore, the extracellular matrix, the cytoskeleton, and membrane proteins may influence distribution of membrane molecules in various ways (Edidin, 2003; Ritchie et al., 2003; Mukherjee and Maxfield, 2004). These results raised a possibility that those nonraft factors are sufficient to generate the GM1 cluster in mouse fibroblasts and that rafts are not involved. Under this conjecture, the result of cholesterol depletion might be attributed to its effect on the actin cytoskeleton (Kwik et al., 2003). However, we observed that manipulation of actin filaments did not affect the GM1 distribution to the extent that can explain the result of cholesterol depletion (Fujita, Cheng, and Fujimoto, unpublished observation). Moreover, chilling is not likely to affect the actin cytoskeleton in the same manner. We thus inferred that preferential partitioning in the ordered membrane is involved at least partially for the generation of GM1 clusters.

Irrespective of the mechanism that facilitates GM1 clustering in living cells, the present result has several implications. First, it has not been easy to understand why cholesterol depletion and chilling could both activate mitogen-activated protein kinase signaling (Furuchi and Anderson, 1998; Kabouridis et al., 2000; Chen and Resh, 2002; Gousset et al., 2002; Magee et al., 2005), because the two manipulations are thought to exert opposite effects on rafts, but the present result on GM1 suggests that the two manipulations may affect the distribution of individual raft-philic molecules in the same manner and could cause a similar outcome. This tenet may be tested directly by probing fine distribution of signaling proteins that reside in the inner leaflet of the cell membrane. Second, cooling on ice has been often used to label cells for microscopy with the tacit supposition that the molecular distribution at an ambient temperature is retained. However, the present results indicated that cooling could change the distribution of some membrane molecules, so data that have been obtained from chilled cells need to be interpreted with caution.

\section{Heterogeneous Clusters of GM1 and GM3}

We observed that both GM1 and GM3 formed clusters susceptible to cholesterol depletion in normal mouse fibroblasts but that the respective clusters were segregated from each other in most cases. In migrating $\mathrm{T}$ cells, a distinct segregation of GM1 and GM3, i.e., GM1 in the uropod and GM3 in the leading edge, was observed (Gomez-Mouton et al., 2001). Our result suggests that GM1 and GM3 also distribute differentially in other cell types, although on a much more minuscule scale. The result also implies that the high distribution density of the two gangliosides has precluded the observation of their segregation by light microscopy in the past.

Biochemical fractions enriched with GM1 and GM3 have been shown to contain different sets of signaling molecules (Iwabuchi et al., 1998; Chigorno et al., 2000). Those fractions are likely to correspond to the GM1 and GM3 domains that we observed, and this would raise two immediate questions. One question is whether the spatial relationship of the two domains would modulate signaling by altering molecular interactions among the signaling proteins. We observed occasional coclustering of GM1 and GM3, which suggests that the two domains could coalesce under certain conditions. It would be interesting to study how coalescence and segregation of those domains are regulated and what is brought about as a result of the domain interactions. The second question is how signaling proteins in the inner leaflet partition to one of the ganglioside domains preferentially. This may be related to the mechanism that generates separate clusters of GM1 and GM3. An intriguing possibility is that GM1 and GM3 are diversified in the ceramide portion and thus favor homologous interactions (Sonnino and Chigorno, 2000; Hakomori Si, 2002). This is not an unrealistic speculation, because glycosyltransferases for the late ganglioside biosynthetic pathways are in the distal Golgi membranes (Lannert et al., 1998), which harbor cholesterol-dependent microdomains, and the probability that a GM3 molecule is processed to become GM1 may depend on the affinity of its ceramide portion to the microdomain where the enzymes exist. As a result, gangliosides remaining as GM3, and those that became GM1, are likely to make homologous clusters more often than heterogeneous gangliosides. Analysis of the ceramide composition of gangliosides is awaited to test the possibility.

Although the underlying mechanism needs to be explored further, our result showed that GM1 and GM3 exist as distinct clusters in the native cell membrane. But the GM1 and GM3 clusters may not be the only microdomains that are affected by cholesterol depletion, and additional microdomains with variable contents are likely to coexist (Marwali et al., 2003; Nagatsuka et al., 2003; Brugger et al., 2004; Kiyokawa et al., 2005). How all those heterogeneous microdomains are formed and how their mutual relationship is controlled warrant further investigation. Hopefully, the present method would help to address these unsolved questions in the near future.

\section{ACKNOWLEDGMENTS}

We thank Drs. Atsuyuki Okabe and Ken-ichiro Shimatani for advice on point pattern analysis, Dr. John Hancock for providing the K-function program, Dr. Masato Umeda for anti-PC antibody, Drs. Akihiro Kusumi and John E. Heuser for helpful discussions, and Kumi Tauchi-Sato and Tetsuo Okumura for assistance. This work was supported by grants-in-aid for scientific research and the 21st Century Center of Excellence Program "Integrated Molecular Medicine for Neuronal and Neoplastic Disorders" of the Ministry of Education, Culture, Sports, Science and Technology of the Japanese Government.

\section{REFERENCES}

Brugger, B., Graham, C., Leibrecht, I., Mombelli, E., Jen, A., Wieland, F., and Morris, R. (2004). The membrane domains occupied by glycosylphosphatidylinositol-anchored prion protein and Thy-1 differ in lipid composition. J. Biol Chem. 279, 7530-7536. 
Chandler, D. E. (1984). Comparison of quick-frozen and chemically fixed sea-urchin eggs: structural evidence that cortical granule exocytosis is preceded by a local increase in membrane mobility. J. Cell Sci. 72, 23-36.

Chen, X., and Resh, M. D. (2002). Cholesterol depletion from the plasma membrane triggers ligand-independent activation of the epidermal growth factor receptor. J. Biol. Chem. 277, 49631-49637.

Chigorno, V., Palestini, P., Sciannamblo, M., Dolo, V., Pavan, A., Tettamanti, G., and Sonnino, S. (2000). Evidence that ganglioside enriched domains are distinct from caveolae in MDCK II and human fibroblast cells in culture. Eur. J. Biochem. 267, 4187-4197.

Dietrich, C., Bagatolli, L. A., Volovyk, Z. N., Thompson, N. L., Levi, M., Jacobson, K., and Gratton, E. (2001a). Lipid rafts reconstituted in model membranes. Biophys. J. 80, 1417-1428.

Dietrich, C., Volovyk, Z. N., Levi, M., Thompson, N. L., and Jacobson, K. (2001b). Partitioning of Thy-1, GM1, and cross-linked phospholipid analogs into lipid rafts reconstituted in supported model membrane monolayers. Proc. Natl. Acad. Sci. USA 98, 10642-10647.

Edidin, M. (2003). The state of lipid rafts: from model membranes to cells. Annu. Rev. Biophys. Biomol. Struct. 32, 257-283.

Friedrichson, T., and Kurzchalia, T. V. (1998). Microdomains of GPI-anchored proteins in living cells revealed by crosslinking. Nature 394, 802-805.

Fujimoto, K. (1995). Freeze-fracture replica electron microscopy combined with SDS digestion for cytochemical labeling of integral membrane proteins. Application to the immunogold labeling of intercellular junctional complexes. J. Cell Sci. 108, 3443-3449

Fujimoto, K., Umeda, M., and Fujimoto, T. (1996). Transmembrane phospholipid distribution revealed by freeze-fracture replica labeling. J. Cell Sci. 109, 2453-2460.

Fujimoto, T. (1996). GPI-anchored proteins, glycosphingolipids, and sphingomyelin are sequestered to caveolae only after crosslinking. J. Histochem Cytochem. 44, 929-941.

Fujimoto, T., and Fujimoto, K. (1997). Metal sandwich method to quick-freeze monolayer cultured cells for freeze-fracture. J. Histochem. Cytochem. 45, 595-598.

Furuchi, T., and Anderson, R. G. (1998). Cholesterol depletion of caveolae causes hyperactivation of extracellular signal-related kinase (ERK). J. Biol. Chem. 273, 21099-21104.

Gaus, K., Gratton, E., Kable, E. P., Jones, A. S., Gelissen, I., Kritharides, L., and Jessup, W. (2003). Visualizing lipid structure and raft domains in living cells with two-photon microscopy. Proc. Natl. Acad. Sci. USA 100, 15554-15559.

Gomez-Mouton, C., Abad, J. L., Mira, E., Lacalle, R. A., Gallardo, E., JimenezBaranda, S., Illa, I., Bernad, A., Manes, S., and Martinez, A. C. (2001). Segregation of leading-edge and uropod components into specific lipid rafts during T cell polarization. Proc. Natl. Acad. Sci. USA 98, 9642-9647.

Gousset, K., Wolkers, W. F., Tsvetkova, N. M., Oliver, A. E., Field, C. L., Walker, N. J., Crowe, J. H., and Tablin, F. (2002). Evidence for a physiological role for membrane rafts in human platelets. J. Cell. Physiol. 190, 117-128.

Hagiwara, A., Fukazawa, Y., Deguchi-Tawarada, M., Ohtsuka, T., and Shigemoto, R. (2005). Differential distribution of release-related proteins in the hippocampal CA3 area as revealed by freeze-fracture replica labeling. J. Comp. Neurol. 489, 195-216.

Hakomori Si, S. I. (2002). Inaugural article: the glycosynapse. Proc. Natl. Acad. Sci. USA 99, 225-232.

Heerklotz, H. (2002). Triton promotes domain formation in lipid raft mixtures. Biophys. J. 83, 2693-2701.

Heider, J. G., and Boyett, R. L. (1978). The picomole determination of free and total cholesterol in cells in culture. J. Lipid Res. 19, 514-518.

Heuser, J. E., Reese, T. S., Dennis, M. J., Jan, Y., Jan, L., and Evans, L. (1979). Synaptic vesicle exocytosis captured by quick freezing and correlated with quantal transmitter release. J. Cell Biol. 81, 275-300.

Iwabuchi, K., Handa, K., and Hakomori, S. (1998). Separation of "glycosphingolipid signaling domain" from caveolin-containing membrane fraction in mouse melanoma B16 cells and its role in cell adhesion coupled with signaling. J. Biol. Chem. 273, 33766-33773.

Jost, P., Brooks, U. J., and Griffith, O. H. (1973). Fluidity of phospholipid bilayers and membranes after exposure to osmium tetroxide and gluteraldehyde. J. Mol. Biol. 76, 313-318.

Kabouridis, P. S., Janzen, J., Magee, A. L., and Ley, S. C. (2000). Cholesterol depletion disrupts lipid rafts and modulates the activity of multiple signaling pathways in T lymphocytes. Eur. J. Immunol. 30, 954-963.
Kenworthy, A. K., Nichols, B. J., Remmert, C. L., Hendrix, G. M., Kumar, M., Zimmerberg, J., and Lippincott-Schwartz, J. (2004). Dynamics of putative raft-associated proteins at the cell surface. J. Cell Biol. 165, 735-746.

Kenworthy, A. K., Petranova, N., and Edidin, M. (2000). High-resolution FRET microscopy of cholera toxin B-subunit and GPI-anchored proteins in cell plasma membranes. Mol. Biol. Cell 11, 1645-1655.

Kiyokawa, E., Baba, T., Otsuka, N., Makino, A., Ohno, S., and Kobayashi, T. (2005). Spatial and functional heterogeneity of sphingolipid-rich membrane domains. J. Biol. Chem. 280, 24072-24084.

Korlach, J., Schwille, P., Webb, W. W., and Feigenson, G. W. (1999). Characterization of lipid bilayer phases by confocal microscopy and fluorescence correlation spectroscopy. Proc. Natl. Acad. Sci. USA 96, 8461-8466.

Kusumi, A., Koyama-Honda, I., and Suzuki, K. (2004). Molecular dynamics and interactions for creation of stimulation-induced stabilized rafts from small unstable steady-state rafts. Traffic 5, 213-230.

Kusumi, A., and Suzuki, K. (2005). Toward understanding the dynamics of membrane-raft-based molecular interactions. Biochim. Biophys. Acta 1746 234-251.

Kusunoki, S., Shimizu, J., Chiba, A., Ugawa, Y., Hitoshi, S., and Kanazawa, I. (1996). Experimental sensory neuropathy induced by sensitization with ganglioside GD1b. Ann. Neurol. 39, 424-431.

Kwik, J., Boyle, S., Fooksman, D., Margolis, L., Sheetz, M. P., and Edidin, M. (2003). Membrane cholesterol, lateral mobility, and the phosphatidylinositol 4,5-bisphosphate-dependent organization of cell actin. Proc. Natl. Acad. Sci. USA 100, 13964-13969.

Lannert, H., Gorgas, K., Meissner, I., Wieland, F. T., and Jeckel, D. (1998). Functional organization of the Golgi apparatus in glycosphingolipid biosynthesis. Lactosylceramide and subsequent glycosphingolipids are formed in the lumen of the late Golgi. J. Biol. Chem. 273, 2939-2946.

Magee, A. I., Adler, J., and Parmryd, I. (2005). Cold-induced coalescence of T-cell plasma membrane microdomains activates signalling pathways. J. Cell Sci. 118, 3141-3151.

Marwali, M. R., Rey-Ladino, J., Dreolini, L., Shaw, D., and Takei, F. (2003). Membrane cholesterol regulates LFA-1 function and lipid raft heterogeneity. Blood 102, 215-222.

Mayor, S., and Rao, M. (2004). Rafts: scale-dependent, active lipid organization at the cell surface. Traffic 5, 231-240.

Mayor, S., Rothberg, K. G., and Maxfield, F. R. (1994). Sequestration of GPI-anchored proteins in caveolae triggered by cross-linking. Science 264 , 1948-1951.

Meder, D., Moreno, M. J., Verkade, P., Vaz, W. L., and Simons, K. (2006). Phase coexistence and connectivity in the apical membrane of polarized epithelial cells. Proc. Natl. Acad. Sci. USA 103, 329-334.

Mukherjee, S., and Maxfield, F. R. (2004). Membrane domains. Annu. Rev. Cell Dev. Biol. 20, 839-866.

Munro, S. (2003). Lipid rafts: elusive or illusive? Cell 115, 377-388.

Nagatsuka, Y. et al. (2003). Carbohydrate-dependent signaling from the phosphatidylglucoside-based microdomain induces granulocytic differentiation of HL60 cells. Proc. Natl. Acad. Sci. USA 100, 7454-7459.

Nam, K. S., Igarashi, K., Umeda, M., and Inoue, K. (1990). Production and characterization of monoclonal antibodies that specifically bind to phosphatidylcholine. Biochim. Biophys. Acta 1046, 89-96.

Nichols, B. J. (2003). GM1-containing lipid rafts are depleted within clathrincoated pits. Curr. Biol. 13, 686-690.

Parton, R. G. (1994). Ultrastructural localization of gangliosides; GM1 is concentrated in caveolae. J. Histochem. Cytochem. 42, 155-166.

Plowman, S. J., Muncke, C., Parton, R. G., and Hancock, J. F. (2005). H-ras, $\mathrm{K}$-ras, and inner plasma membrane raft proteins operate in nanoclusters with differential dependence on the actin cytoskeleton. Proc. Natl. Acad. Sci. USA $102,15500-15505$

Pralle, A., Keller, P., Florin, E. L., Simons, K., and Horber, J. K. (2000) Sphingolipid-cholesterol rafts diffuse as small entities in the plasma membrane of mammalian cells. J. Cell Biol. 148, 997-1008.

Prior, I. A., Muncke, C., Parton, R. G., and Hancock, J. F. (2003). Direct visualization of Ras proteins in spatially distinct cell surface microdomains. J. Cell Biol. 160, 165-170.

Ripley, B. D. (1977). Modeling spatial patterns. J. R. Stat. Soc. Ser. B 39, $172-212$

Ripley, B. D. (1979). Tests of randomness for spatial point patterns. J. R. Stat. Soc. Ser. B 41, 368-374. 
Ritchie, K., Iino, R., Fujiwara, T., Murase, K., and Kusumi, A. (2003). The fence and picket structure of the plasma membrane of live cells as revealed by single molecule techniques. Mol. Membr. Biol. 20, 13-18.

Schnitzer, J. E., McIntosh, D. P., Dvorak, A. M., Liu, J., and Oh, P. (1995). Separation of caveolae from associated microdomains of GPI-anchored proteins. Science 269, 1435-1439.

Schwarzmann, G., Hoffmann-Bleihauer, P., Schubert, J., Sandhoff, K., and Marsh, D. (1983). Incorporation of ganglioside analogues into fibroblast cell membranes. A spin-label study. Biochemistry 22, 5041-5048.

Sharma, P., Varma, R., Sarasij, R. C., Ira, Gousset, K., Krishnamoorthy, G., Rao, M., and Mayor, S. (2004). Nanoscale organization of multiple GPIanchored proteins in living cell membranes. Cell 116, 577-589.

Simons, K., and Ikonen, E. (1997). Functional rafts in cell membranes. Nature $387,569-572$.

Simons, M., Friedrichson, T., Schulz, J. B., Pitto, M., Masserini, M., and Kurzchalia, T. V. (1999). Exogenous administration of gangliosides displaces GPI-anchored proteins from lipid microdomains in living cells. Mol. Biol. Cell $10,3187-3196$

Sonnino, S., and Chigorno, V. (2000). Ganglioside molecular species containing C18- and C20-sphingosine in mammalian nervous tissues and neuronal cell cultures. Biochim. Biophys. Acta 1469, 63-77.

Takamiya, K. et al. (1996). Mice with disrupted GM2/GD2 synthase gene lack complex gangliosides but exhibit only subtle defects in their nervous system. Proc. Natl. Acad. Sci. USA 93, 10662-10667.
Taki, T., and Ishikawa, D. (1997). TLC blotting: application to microscale analysis of lipids and as a new approach to lipid-protein interaction. Anal Biochem. 251, 135-143.

Thompson, T. E., Allietta, M., Brown, R. E., Johnson, M. L., and Tillack, T. W. (1985). Organization of ganglioside GM1 in phosphatidylcholine bilayers. Biochim. Biophys. Acta 817, 229-237.

Varma, R., and Mayor, S. (1998). GPI-anchored proteins are organized in submicron domains at the cell surface. Nature 394, 798-801.

Vie, V., Van Mau, N., Lesniewska, E., Goudonnet, J. P., Heitz, F., and Le Grimelle, C. (1998). Distribution of ganglioside GM1 between two-component, two-phase phosphatidylcholine monolayers. Langmuir 14, 4754-4783.

Wang, T. Y., and Silvius, J. R. (2003). Sphingolipid partitioning into ordered domains in cholesterol-free and cholesterol-containing lipid bilayers. Biophys. J. $84,367-378$.

Williams, M. A., and McCluer, R. H. (1980). The use of Sep-Pak C18 cartridges during the isolation of gangliosides. J. Neurochem. 35, 266-269.

Yuan, C., Furlong, J., Burgos, P., and Johnston, L. J. (2002). The size of lipid rafts: an atomic force microscopy study of ganglioside GM1 domains in sphingomyelin/DOPC/cholesterol membranes. Biophys. J. 82, 2526-2535.

Yuan, C., and Johnston, L. J. (2000). Distribution of ganglioside GM1 in L-alpha-dipalmitoylphosphatidylcholine/cholesterol monolayers: a model for lipid rafts. Biophys. J. 79, 2768-2781.

Zhou, X., and Arthur, G. (1992). Improved procedures for the determination of lipid phosphorus by malachite green. J. Lipid Res. 33, 1233-1236. 